\title{
Theologie und Kommunikation
}

\author{
W. G. Tillmans
}

Dieser Artikel ${ }^{1}$ kann nur global und generalisierend sein. Von vielen Disziplinen und Strömungen der Theologie wie der Kommunikationswissenschaft muß ich absehen. Die Ökumene und das Gespräch mit anderen Religionen können ebenso wenig zur Sprache gebracht werden. Was übrig bleibt, ist eine allgemeine Betrachtung aus dem Blickwinkel der systematischen Theologie mit der Absicht, einige Linien darzustellen, die richtungsweisend im Gespräch zwischen Theologie und Kommunikationswissenschaft sind.

Das Gespräch zwischen Fachleuten auf dem Gebiet der Theologie und dem der Kommunikationswissenschaft ist in jüngster Zeit intensiver geworden. Hierzu haben verschiedene Faktoren beigetragen. In erster Linie muß hier die schnelle Entwicklung der Massenmedien genannt werden. Nach der Zeitung, dem Film und dem Radio ist inzwischen das Fernsehen mit Video, Video-Text und Bildschirmtext gänzlich in die kulturelle Wirklichkeit vorgedrungen. Die Welt wird kommunikativ verkleinert. Jeden Morgen und Abend wird uns, selektiv und geordnet, die Welt aufgetischt. Durch diese Information wird der Empfänger über große Entfernungen in Geschehnisse und Entwicklungen mit einbezogen, die sichtlich auch Bedeutung für unser Leben hier haben. In den verschiedensten Darstellungsformen werden Werte und Perspektiven vermittelt, die den (künstlichen) Horizont unseres täglichen Lebens formen. Dieser soziale und kulturelle Einfluß verlangt unsere Aufmerksamkeit: Wie müssen wir religiös, ethisch und politisch damit umgehen? Darüber hinaus scheint bei den Kirchen, innerhalb des heutigen offenen Miteinanders der Menschen, ein wachsendes Verlangen nach einer guten Kommunikationsstrategie zu entstehen, sowohl für das Gespräch innerhalb der Kirche wie auch für die öffentliche Kommunikation nach außen. Unmittelbar hiermit verbunden ist die Notwendigkeit einer guten kommunikativen Ausbildung der kirchlichen Mitarbeiter.

Aus der Sicht der Theologie selbst ist noch ein anderer wichtiger Faktor zu erwähnen: Kommunikation hat zu tun mit dem Miteinander von Gott und Mensch und dem daraus folgenden Umgang der Menschen untereinander. Indem die Theologie hierüber nachdenkt, fragt sie auch nach den Möglichkeiten und Aufgaben ihres eigenen kommunikativen Funktionierens innerhalb der Glaubensgemeinschaft und der sie umgebenden kulturell-gesellschaftlichen Welt.

Hier bin ich ins Zentrum meiner Betrachtungen gelangt. Ausgehend von meiner theologischen Erfahrung will ich zur folgenden Frage Stellung nehmen: Welche Bilder und Ideen habe ich von Kommunikation, und wie bringen mich diese Auffassungen ins Gespräch mit der Kommunikationswissenschaft? Dabei möchte ich keine systematische Übersicht geben, ausgehend von dem Begriff der Kommunikation, sondern Kommunikation als Erscheinung und Idee auffassen, die eine sehr wichtige Rolle in der theologischen Reflexion spielt. Hieraus ergibt sich denn auch der Aufbau meines Artikels. Zunächst folgen zwei Abschnitte über die Kommunikation als Gegenstand

Prof. Dr. W. G. Tillmans ist seit 1980 Professor für Systematische Theologie an der Katholisch-Theologischen Hochschule in Amsterdam und seit 1985 Rektor dieses Instituts. 
theologischen Denkens, danach betrachte ich das Gespräch mit der Kommunikationswissenschaft und schließe, indem ich einige Linien aufzeige zur Kommunikation in der Theologie selbst und zur theologischen Ausbildung. Es geht hierbei um einen komplexen Sachverhalt. Die Aufhellung läßt viele Denkmodelle zu. Ich wähle die Form der Annäherung, die sich vor allem auf das sakramentale Modell stützt.

\section{Die heilsgeschichtliche Fortfübrung der Offenbarung als Kommunikation}

In den letzten Jahrhunderten vollzog sich die Verkündigung und Lehre der Kirche vorwiegend in recht abstrakten Vorstellungen und Konzepten. Dieser »Wahrheitspositivismus « ist beendet: Durch den Anschluß an das existentiell-personalistische Denken (mit besonderem Nachdruck auf der Leiblichkeit), durch eine erneuerte Schriftlesung (mit dem Sinn für den historischen Prozeß der Verbindung Gottes mit den Menschen) und durch mehr Kenntnis der alten Tradition (mit ihrer typisch symbolisierenden Art zu sprechen) ist die Theologie sich der Vielfalt und der Variation der Sprachformen, durch die die Offenbarung als immer neue und lebendige Gegenwart weitergegeben wird, bewußt. Resultat dieser Entwicklung ist, daß man inzwischen nicht zuletzt durch den Einfluß des Zweiten Vatikanums - die Offenbarung nach dem Modell der Kommunikation versteht. Ich kann hier nur einige der Elemente dieses Gedankenganges erwähnen.

Die Heilige Schrift kann als eine Geschichte gelesen werden, die beim andächtigen $\mathrm{Zu}$ hörer Einsicht und Betroffenheit weckt. Es ist immer eine gute Geschichte im doppelten Sinne dieses Wortes: Es geht stets um die Relation zwischen dem Gewöhnlichen und dem Außergewöhnlichen, zwischen dem Bekannten und dem Unerwarteten. In dieser Relation können Glauben und Gehorsam geboren und genährt werden. Das bekannteste Beispiel solchen narrativen Sprechens ist die Parabel, die auch in Jesu Predigten einen beliebten Platz einnimmt. Durch die Benutzung von Charakteren, Situationen und Symbolen verlockt die Parabel zum Hinhören. Am Ende stößt der Leser mit einem Schock auf unerwartete Fragen über sein eigenes Leben. Bevor er es merkt, ist er gänzlich in die Geschichte verwickelt. So kann auch das Leben Jesu als eine Parabel erzählt werden. Die Geschichte ruft Einsichten hervor, aber am Ende zeigt sich, daß Gott selbst den Erniedrigten und Verworfenen unterstützt und uns so die Frage nach der Wahl, nach der Entscheidung stellt. Die gleiche narrative Vermittlung sehen wir in der Prophetie oder - mehr in der Sphäre der biblischen Weisheit im griechischen Denken - in dem Wort, das fleischwerdend Gottes Transzendenz als ordnende Liebe inmitten der Menschen einpflanzt. So sehen wir, daß die Offenbarung sich vollzieht (bis in unsere Zeit hinein) als ein Gespräch von und mit Menschen in ihrer Zeit und auf ihrem Platz, kurz in einer konkreten Situation. Gott interveniert in der Geschichte in und durch Menschen. Er erzeugt Verstehen und Antworten. Daher kommt es, daß die dogmatische Konstitution »Dei Verbum« des Zweiten Vatikanischen Konzils die Offenbarung in der Terminologie der Kommunikation beschreiben kann: "Durch die göttliche Offenbarung wollte Gott sich selbst und die ewigen Entscheidungen seines Willens über das Heil der Menschen kundtun (manifestare) und mitteilen (communicare) (D.V. 6). Es geht also nicht allein um das Mitteilen von Wahrheiten (vgl. Vatikanum I: revelare), sondern um das Geben von sich selbst. Dadurch ist die Offenbarung eine persönliche Kommunikation, ein Dialog zwischen Gott und Mensch: Gott will eine „Communio“, ein Zusammensein mit den Menschen. Das überschreitet freilich das Informationsmodell. Die fortschreitende Offenbarungseinheit hat als kommunikatives Geschehen seinen Höhepunkt in Jesus Christus. Durch seine ganze Person kommt Gottes Hingabe zu Wort. Durch Wort 
Wort. Durch Wort und Werk, Zeichen und Wunder, durch Tod und Auferstehung und schließlich durch die Gabe des Geistes ist er ein enthüllendes Zeugnis von der Tatsache, daß Gott mit uns ist (D.V. 2).

Die nachkonziliare Instruktion "Communio et progressio * ist eine Weiterausarbeitung dieses kommunikativen Offenbarungsmodells; dieses Modell selbst wird als eine Richtlinie der menschlichen Kommunikation benutzt. Ziel jeder Kommunikation ist die christliche "Communio«. Um Gottes Kommunikationswillen zu manifestieren, ist Jesus Christus der definitive und vollmächtige Kommunikator ("Perfectus Communicator «, C.P. 11). Er begab sich selbst in eine menschliche Communio und öffnete für die Menschen so den Zugang zu der göttlichen Communio. Zu diesem Kommunikationsmodell der Offenbarung möchte ich nun einige positive und negative Anmerkungen hinzufügen.

\subsection{Einige Anmerkungen mehr positiver Art}

Das theologische Sprechen ist ein analoges Sprechen, das bedeutet: Menschliche Gegebenheiten in der Kommunikation werden als Bild genutzt, um Gottes Umgang mit den Menschen zu verdeutlichen. Dieses Sprechen ist lediglich eine Spur, ein Richtungspfeil zum Geheimnis Gottes in seinem Handeln mit den Menschen.

Man kann also nicht einfach die Eigenschaften und Charakteristika menschlicher Kommunikation auf Gott selbst übertragen: Das Transzendente übersteigt nun einmal unser Auffassungsvermögen.

Umgekehrt gibt dieses Modell verschiedene Einsichten für das menschliche Handeln innerhalb des Offenbarungsgeschehens. So scheint die Offenbarung dauernd verbunden zu sein mit ihrer Aufnahme, der menschlichen Rezeption. Wahrheit besteht nur, wenn sie verstanden wird. Man denke hierbei an das Interesse der sogenannten pragmatischen Exegese, die jeden Nachdruck legt auf die Aufnahme der Frohbotschaft durch den Hörer des Textes. Ihrem Wesen nach ist die Offenbarung sowohl ein menschliches als auch ein göttliches Geschehen, obwohl der antwortende Glaube immer die Initiative in Gott sehen wird. Offenbarung geschieht in und durch Menschen in einer unauflöslichen Verwobenheit von Hören und Antworten. Es sind immer Menschen, inklusive Jesus Christus, die Gottes Gegenwart bezeugen.

Die Botschaft Gottes ist also mit der menschlichen Antwort so verbunden, daß sich in stets verschiedenen Situationen, auf stets verschiedene Art und Weise diese Offenbarung sehen und hören läßt. Dies ist das Konzept der "Menschwerdung «, die die Offenbarung immer aktuell sein läßt. Offenbarung, auch im Verlauf der Tradition (Überlieferung), ist also immer eine "gute Geschichte«. Das meint: Sie schließt sich immer neuen Lebenseinsichten und Lebensgewohnheiten und stets neuen Hörern an. Dies besagt ferner, daß die Menschen auch in der Lage sein müßten, das Wort zu hören, zu verstehen und sich danach zu orientieren. Die Theologie verweist hierbei auf die Schöpfungsgeschichte, in der alles Geschaffene auf Gott bezogen ist. Außerdem ist der Mensch als »Abbild Gottes « fähig zu transzendierender Kommunikation, was bedeutet, daß er sich selbst und seine Welt ausdrücken kann als ein endloses Geheimnis, das die Begrenztheit der vorläufigen Einsicht und des vorläufigen Zustandes übersteigt. Die Beschreibung der Offenbarung als Gottes Hingabe bedeutet eine Überwindung der sachlichen Perspektive. Die Kommunikation von Gott und Mensch betrifft nicht eine Handelstransaktion, den informativen Austausch von Absichten, sondern 
einen persönlichen Dialog, in dem jemand völlig an dem anderen als anderem interessiert ist. Es geht hierbei also um »extatische " Selbstverwirklichung: Ich bin erst ich selbst, wenn ich weg bin vom anderen.

Schließlich gibt dieses Kommunikationsmodell an, daß der Umgang mit Gott und deshalb auch den Menschen untereinander viel mehr umfaßt als distanziertes rationales Sprechen. Wenn die Tradition die Offenbarung weiterführt, ist damit eine Übertragung von Worten und Taten gemeint, eine verbale und nonverbale Kommunikation, in der die ganze Leiblichkeit einbezogen ist. In der Liturgie, in der Katechese, kurzum in der gesamten christlichen Kultur geht es darum, daß der ganze Mensch in all seinen Facetten angesprochen wird, damit der ganze Mensch in all seinen $\mathrm{Fa}_{\mathrm{a}}$ cetten antworten kann. Die Sprache der Offenbarung ist deshalb zunächst relational und symbolisch. Es ist die Rede von einem Verhältnis, das sich verweisend selbst transzendiert und von einer Transparenz unter Menschen, die Gott selbst präsent sein läßt.

\subsection{Einige Anmerkungen mehr negativer Art}

Das kirchliche und dialogische Sprechen beachtet nicht immer den ihm innewohnenden tastend-analogen Charakter. Darum wirkt dieses Sprechen hier und da imperialistisch, namentlich wenn dieses Sprechen die eschatologische Dimension vergißt und sich in einem geschlossenen Perfektionismus - als Botschaft - von der Welt unterscheiden will. $\mathrm{Zu}$ recht protestieren die Humanwissenschaften und auch die Kommunikationswissenschaften dagegen. Es kann keine Rede davon sein, daß der Glaube oder das Kirchengeschehen sich dieser Welt entziehen muß. Gerade vom inkarnatorischen Offenbarungsprinzip her muß man sagen, daß es ein Geschehen der Geschichte ist in unserer Welt und deshalb, ebenso wie andere Dinge, zugänglich ist für empirische und sachliche Betrachtungen.

Um nur ein Beispiel zu geben: Man kann Jesus Christus "Perfectus Communicator « nennen, aber man darf dann nicht vergessen, daß man innerhalb einer Glaubensperspektive spricht, zu der Tod und Auferstehung gehören. Jesus ist ein perfekter Kommunikator für die, die ihn in diesem Glauben erfahren und sehen. Als empirische Wirklichkeit war die Kommunikation Jesu sicher nicht erfolgreich; seine Hingabe an die Botschaft des Reiches Gottes hatte einen kenotischen, einen sich selbst zerstörenden Charakter. Bei seinen Jüngern und Anhängern endete seine Kommunikation deutlich in einem Mißerfolg. Das meiste Verständnis fand er bei Außenseitern, bei Bettlern, Prostituierten, Witwen und Kindern.

Auch die Neuigkeitskriterien der frohen Botschaft sind anders als die unseren. Deren »Prominente« sind nicht die Reichen und Mächtigen, sondern die Armen und Ohnmächtigen; Tatsachen werden ersetzt durch Mysterien, und zeitlos objektive Information wird zum Geschehen von hier und heute, von dem man direkt betroffen ist. So scheint es, daß man das Modell der Kommunikation nicht ohne Anpassung auf das theologische Sprechen übertragen kann.

Schließlich ist da noch ein Element, das in diesem Zusammenhang eine Anmerkung verdient, nämlich: Das äußerliche Wort der Botschaft kann nicht ohne innerliches Wort sein. Die fortschreitende christliche Kommunikation kann nicht ohne Beistand des Heiligen Geistes sein. Dieser Unterschied zwischen äußerlichem und innerlichem Wort geht zurück auf den christlichen Gedanken, daß der Glaube selbst und die ganze 
damit verbundene Weisheit eine Gabe ist, deren Ursache nicht die effiziente Kommunikation des Evangeliums sein kann. Dennoch: Diese Kommunikation ist als Vermittlerin unentbehrlich, um die einmalige Mittlerschaft Christi in dieser Welt zur Geltung kommen zu lassen.

In der Theologie ist es deshalb nicht möglich, von einem "außerhalb « bestehenden Sender als Gegenpol innerhalb der Glaubenskommunikation zu sprechen. Auch wenn man - zu recht - den Nachdruck auf den konkret-partikulären Charakter des Lebensgeheimnisses Christi ("Mysteria carnis Christi«) legt, um dem Glaubensleben Richtung zu geben, wird es nicht um eine historisch-distanzierte Betrachtung gehen können, sondern um eine aktuelle Teilnahme, welche nicht ohne den Geist geschehen kann. In welcher Kommunikation werden die Evangelien übrigens zu einem aktuell gültigen Evangelium, das den Menschen befreiend erhebt, um dem Reich Gottes zuversichtlich entgegenzugehen?

\section{Das kirchliche Leben als ein sakramental-kommunikativer Prozeß}

Die letzte Frage führt uns hinein in das Feld des kirchlichen Lebens (auch in seinem Verhältnis zur Welt), das gleichfalls stark verbunden ist mit dem Begriff der Kommunikation. Man kann hierbei unterscheiden $z$ wischen dem mehr innerlichen und verborgenen Aspekt des persönlichen Glaubenslebens und dem mehr äußerlichen und öffentlichen Aspekt des kollektiven Glaubenslebens sowohl innerhalb der Glaubensgemeinschaft als auch im Umgang dieser Gemeinschaft mit der sie umgebenden Welt.

Eines der großen Geheimnisse im christlichen Leben ist die Vereinigung des Gläubigen mit Jesus Christus als dem einzigartigen Mittler. Die Schrift kennt viele Bilder, um dieses "Sein in Christus « zu verdeutlichen. Herauszuheben ist hierbei die Brotrede im Johannesevangelium (Joh 6), worin Glauben mit dem Essen verbunden wird. Die Väter, unter ihnen Augustinus und die frühe Scholastik, sprechen in Kommentaren zu diesem Text von einer "Manducatio Spiritualis «, einer geistlichen Speise, oder von einer »Communio Spiritualis «. Bis zur Hochscholastik betrachtet man diese geistliche Communio mit Christus als das fundamentalste und umfassendste Kennzeichen des Glaubenslebens. Die persönliche Einheit mit Christus in Glaube und Liebe ist dann das Umfeld, worin die Sakramente mit ihrer Zeichenkraft wirken: Sie manifestieren innerhalb der Glaubensgemeinschaft genau das Gnadenleben, das selbstverständlich auch als ein außerhalb der Sakramente Existierendes gedacht wird. Als die Sakramente jedoch in ihrer Siebenzahl einzeln herausgegriffen werden und - als instrumentelle Heilsmittel - ihnen eine eigene Gnadenwirkung zugeschrieben wird, verschwindet diese alte Selbstverständlichkeit. Ab dem 14. Jahrhundert (Devotio Moderna) wird in zunehmendem Maße die "geistliche "Communio und die damit verbundene geistliche Kommunikation nicht mehr als das Fundament des christlichen Lebens angesehen. Es wird eine eigene, eher marginale Devotion.

Im Osten hat die geistige Kommunikation mit Christus ihren fundamentalen Wert beibehalten. Im Westen blieb der Begriff "Communio* und "Kommunikation * weiter im spirituellen Schrifttum bestehen. So braucht Johannes vom Kreuz im 16. Jahrhundert den Begriff Kommunikation noch als einen einfachen technischen Terminus, um den Umgang der Seele mit Gott anzugeben: Eine wachsende zweiseitige Mitteilung auf dem Weg der gläubigen Nachfolge, die sich verwirklicht in der vollendeten mystischen "Communio « oder Vereinigung. 
Es ist wichtig, auf das Bestehen dieser Tradition hinzuweisen. Es geht hierbei natürlich um mehr als um das, was man die »intrapersonale Kommunikation « nennt. In dieser Tradition wird der Gläubige gekennzeichnet durch einen fortdauernden und emotionalen Dialog (mit Christus und) mit Gott. Von Bedeutung ist nicht allein, daß hierin ein enormes kulturelles Erbgut von gläubiger Weisheit im Verkehr mit Gott verborgen liegt, sondern auch, daß dieser persönliche Umgang mit Gott stets anwesend ist im Gespräch mit anderen, und zwar mit Menschen, die ich hierzu anleiten könnte: im Gruppenverband und oft selbst in größeren Glaubensgemeinschaften. Diese Kultur der Kommunikation mit Gott und miteinander über genau dieses Fundament des christlichen Lebens ist ziemlich verdörrt und vergessen. Erst in unserer Zeit wird von kommunikativer Spiritualität wieder Gebrauch gemacht, z. B. in Gesprächsgruppen, Gebetsübungen, Textlesungen im Gruppenverband.

Nur in Ausnahmefällen beschäftigt sich die akademische Theologie mit dem einen oder anderen, während doch schon lange von gottesdienstpsychologischer und soziologischer Seite auf die Bedeutung einer Spiritualitätskultur hingewiesen wurde. Es dürfte deutlich sein, daß gerade auf diesem Niveau die Symbolik von Bild und Sprache geformt wird, eine Symbolik, die als »communion mythique« oder als »Metakommunikation " den Hintergrund und den Boden aller weiteren christlichen Kommunikation dieser Welt darstellt. $\mathrm{Zu}$ lange hat die offizielle Kirche und die offizielle Theologie mit ihrer technischen Sprache die christliche Kommunikation beherrscht. Man könnte sogar sagen, daß die Einbahnstraße der offiziellen Glaubensäußerung zuviel aus der Alltagssprache entnommen hat.

Ich bin davon überzeugt, daß die Theologie in dieser Tradition nuancierte Sprachspiele und Kommunikationsformen finden kann, die als christlicher Reichtum das Gespräch mit den Kommunikationsfachleuten befruchten können.

Doch ist der Begriff Kommunikation (Communio) längst wieder auf anderem Wege in die theologische Systematik eingedrungen. Diese Wende kommt in den Dokumenten des II. Vaticanums zum Tragen. Hierbei sind zwei Entwicklungen von Bedeutung. Zum einen hat sich die Theologie im letzten Jahrhundert selbst intensiv mit der Identität der Kirche in und für die Welt beschäftigt. Zum zweiten ist vor allem in der Theologie nach dem II. Vaticanum eine erneuerte Einsicht in die Sakramente gewachsen. Sie werden jetzt wieder betrachtet als Glaubenszeichen (also nicht mehr als bloß externe Instrumente, um Gnade zu erlangen) und so aus ihrer Isoliertheit geholt. Die Sakramente werden als wichtige Momente in den einen allgemeinen Begriff von $\gg \mathrm{Sa}-$ kramentalität« eingebracht. Hiermit meint man, daß das ganze Glaubensleben Gestalt gewinnt im Umgang mit den Dingen und den Mitmenschen; in Sinn, Sprache und Handeln verleiht der Glaube allem Geschaffenen eine typische Transparenz, die Gott in dieser Welt präsent macht.

Dieser verbreitete Sakramentsbegriff wird nun angepaßt an das Ganze der Glaubensgemeinschaft, an die Kirche, die Christus (als das Ursakrament) in dieser Welt gegenwärtig hält. Dadurch, daß Kirche und Sakrament zusammengeführt wurden, wird das Glaubensleben als ein kommunikativer Prozeß beschrieben. Hierzu kann man zwei Anmerkungen machen:

Zunächst wird die Bedeutung der vorgegebenen Glaubensgemeinschaft für den individuellen Gläubigen dargelegt. Zum zweiten wird die Glaubenssprache selbst jetzt über die dogmatische Sprachform hinaus ausgeweitet zum Rhetorischen, zum Erzählen- 
den, zum Leiblichen und zum Praktischen, kurz zum Symbolischen als der umfassendsten Sprachform des gläubigen Umgangs mit der Welt.

In der Konstitution über die Kirche "Lumen Gentium « wird die Kirche Sakrament genannt: Zeichen und Werkzeug zum Dienst am Heil der Welt. Die Kirche selbst ist Welt, aber dann ein Platz in dieser Welt, wo Gottes kommunikative Absicht mit den Menschen manifest wird. In ihrer Erscheinung läßt die Kirche sehen, wohin das ganze Menschheitsgeschlecht unterwegs ist. Die Kirche ist nicht identisch mit dem Reich Gottes, aber der Geist kann in ihr, wenn auch nicht exklusiv, die intertrinitäre Communio spüren lassen als Zeichen der heilsgeschichtlichen universellen Zukunft. Dadurch kennzeichnet das Konzil die Heilsgeschichte als einen kommunikativen Prozeß. Einst werden alle Menschen mit Christus vereinigt sein in der Communio mit Gott selbst.

In dieser Perspektive bekommt Kommunikation eine besondere Tiefe. Die Kirche, alle Gläubigen, ja alle Menschen sind in der Lage, sich selbst zu geben, mit einer Transparenz, die Gott sehen läßt. Die menschliche Welt wird verwiesen auf eine kommunikative Verwirklichung des Tiefsten im Schöpfungs- und Bundesgedanken: Die enthüllende Anwesenheit des Transzendenten im Geheimnis des Menschen und seiner ganzen geschaffenen Wirklichkeit.

Wenn man verschiedene Thomas-Lexika unter diesem Gesichtspunkt aufschlägt, sieht man, daß die Begriffe "Communicatio « und »communicare « mehr Raum für sich in Anspruch nehmen als der Begriff »Communio «. Seit dem Vaticanum II aber ist der Communio-Gedanke ins Zentrum gerückt, auch als Übersetzung des alten "koinonia " (das ist das Ideal der universellen Einheit zwischen den Ortskirchen). Bei "Communio* wird außerdem immer an die Kommunikation innerhalb der Trinität gedacht: die göttliche Bewegung als Anfang und Ende der Heilsgeschichte. Diese göttliche "Communio« ist die Gabe des Geistes an (wieder nicht exklusiv) die Kirche als Glaubensgemeinschaft. Diese Gabe in der Zeit und in der Welt wird theologisch am ehesten begriffen als Sakrament.

Kommunikativ ist "Sakrament " nicht eines der am stärksten sprechenden Bilder der Kirche, aber aufgrund der vielen Möglichkeiten wird dieser Begriff in der Systematik am meisten gebraucht. Will man ihn kommunikativ verwendbar machen, was einige Theologen schon versucht haben, dann kann man am besten ausgehen von der Eucharistie, als der typisch gläubigen Weise des Miteinanderumgehens. Die Gemeinschaft ist dann nach innen gekehrt um ihren Herrn und im Gespräch mit dem Vater; sie kommt zum eigentlichen Geheimnis und übersteigt damit alle Selbstbeurteilung in der Auseinandersetzung mit der Welt. Die Initiationssakramente kann man als Ausgangspunkt nehmen, wenn man das Gespräch der Glaubensgemeinschaft mit der sie umringenden Kultur beschreiben will. In der Vorbereitung und während der Feier der Taufe tritt die Kirche immer in das Gespräch mit der Außenwelt ein, die sie einladen will zu Christus und zu sich selbst. In diesem Gespräch gibt sie sich selbst eine Identität und ein Gesicht, das ein erkennbares Versprechen sein will. Insgesamt haben wir es hier zu tun mit „Communio « als einem Idealbild von kirchlicher Kommunikation. Kann man damit arbeiten?

\section{Kommunikation als christliches Ideal im Gespräch mit der Kommunikationswis- senschaft}

Theologisches Sprechen neigt schnell zu weitgehender Abstraktion: Ideen über Ideen. In der Tat beschäftigt sich all das Vorhergehende mit einem Ideal, das sich für den 
Glauben auch anbietet als Realität, die einmal vollständig manifest werden wird; es betrifft ein Ideal, das in Richtung der eschatologischen Erwartung geht und darum nie triumphalistisch oder perfektionistisch verbunden werden kann mit der aktuellkonkreten Situation des kirchlichen Lebens innerhalb dieser Welt. Doch ist die Erwartung, die seit dem II. Vaticanum in den Begriff Kommunikation investiert wurde, nicht aus der Luft gegriffen, im Gegenteil. Es ist ein Modell der Hoffnung, das entstanden ist aus dem ernsthaften Lesen der Zeichen unserer Zeit. Es ist eine Einstimmung in die theoretische und praktische Einsicht, daß Menschen einander nötig haben, um zu leben; daß die Welt einen universellen Mangel an Solidarität hat und daß schließlich auf religiösem Niveau Gott sich selbst zu manifestieren scheint (über alle sachlichen und wertenden Transaktionen hinaus) als endlose Selbsthingabe zwischen den Menschen. Das ausdrückliche Streben, das Glaubensleben und das kirchliche Leben zuzuspitzen auf die Verbesserung und Vertiefung von Kommunikation als gegenwärtige Form der Heilserwartung, hat theologisch gesehen dann auch gute Aussichten.

Theologisch haben wir besser erkannt als jemals zuvor, daß die Kirche und die Gläubigen sowohl beim Empfang der Botschaft als auch in der Praxis der Nachfolge mitten im Leben stehen und daß der Prozeß der Heilsgeschichte verwoben ist mit menschlichen und wirtschaftlichen Interaktionen. Das Gespräch mit den Humanwissenschaften ist schon eine gute Tradition geworden. Jetzt deutet sich ein neuer Gesprächspartner an: Der Kommunikationsfachmann.

Wie in der Theologie sind auf dem Gebiet der Kommunikation viele wissenschaftliche Disziplinen, Methoden, Arbeitsformen, Theorien und Modelle entstanden. Neben dem beherrschenden empirisch-instrumentalen Zweig gibt es auch die anthropologisch-kulturellen Richtungen, die die Kommunikation (namentlich die Massenmedien) untersuchen im Hinblick auf universale Bewußtwerdung und Solidarität. Es gibt inzwischen auch Theorien wie die $» s a l v a t i o n-t h r o u g h-c o m m u n i c a t i o n *$ die $»$ herrschaftsfreie « Kommunikation (J. Habermas); und im Spiel ist ebenfalls, über die "New World Information and Communication Order", die kommunikative Befreiungsbewegung der Dritten Welt. Diese und andere Theorien und Bewegungen sprechen die Theologie direkt an in Fragen des Austausches und der Zusammenarbeit.

Das interdisziplinäre Studium selbst ist erst in jüngster Zeit in Gang gekommen und wird noch viel Anstrengung abverlangen. Erst wenige Theologen sind einigermaßen vertraut mit der (Fach)sprache und den Denkmodellen der Kommunikationswissenschaft. Es ist dadurch auch unvermeidlich, daß hier und da Fehler vorkommen, indem allzu leichtfertig Termini und Modelle verwechselt werden. Die Begriffe, die in der Theologie funktionieren, bleiben - so objektiv sie auch konzeptualisiert worden sind - zum größten Teil analoger Art, Spuren auf dem Geheimnis Gottes und des Menschen. Sie tragen immer eine nicht zu leugnende eschatologische Erwartung in sich. Positiv ist, daß die Theologie ein besonderes Gespür hat für die transzendentale Dimension in der menschlichen Kommunikation. Sie verweist stets auf das Ideal Selbsthingabe: Sowohl intrapersonal als auch interpersonal geht es dann um eine offene Wirklichkeitserfahrung. Die Wirklichkeit wird hier gesehen als ein Geheimnis innerhalb und außerhalb des Menschen, das sich stets enthüllend weiter den menschlichen Begriffen und dem menschlichen Beherrschen entzieht. Es scheint stets mehr Wirklichkeit zu geben, als festgestellt worden ist. Das Selbst des Menschen und das Selbst der Welt sind wie ein Fenster nach Gottes einladender Anwesenheit. 
Man deute meine Betrachtung nicht als die Abweisung aller instrumental ausgerichteten Studien. Für die Praxis der kirchlichen Kommunikation kann im Hinblick auf die Effektivität, noch sehr viel gelernt werden. Ich würde es allerdings bedauern, wenn wo gerade jetzt das instrumentelle Denken in der Glaubensgemeinschaft langsam überwunden wird - schließlich eine Kommunikation die Überhand gewänne, die sich selbst nur - und dabei sehr raffiniert - im Horizont von Zweck und Mittel begreift. Letzteres geschieht auch im kirchlich-theologischen Umgang mit den Massenmedien als Mittel zur Verkündigung und Selbstpräsentation. In dieser allzu schnellen Identifikation der Offenbarung mit effektiver Kommunikation drohen der Glaube und die Kirche selbst zu Opfern zu werden: Man hat noch nicht erkannt, daß durch diese Art der Präsentation die Gefahr der Verzettelung in der Kommunikationswelt droht, einer Kommunikationswelt, die dem Christentum und der Kirche bereits eine (sterotype) Identität zugeschrieben hat. Möchte man diese denn nur naiv festigen?

\section{Kommunikation in der Theologie selbst}

Das christliche Kommunikationsideal betrifft auch die Theologie selbst. Kommunikation ist nicht das Vorrecht einzelner Spezialisten, sondern eine fundamentale Dimension des theologischen Sprechens. Es ist demnach auch nicht gut, daß in der theologischen Ausbildung wie in der theologischen Weiterbildung Studenten zunächst eigentlich nur informiert und erst später als eine Art Anhängsel besonders unterrichtet werden in der Wissenschaft und Praxis der Kommunikation. Kommunikation kann nicht als ein eigenes Fach neben die inhaltlichen Fächer eingebaut werden, z. B. bei der praktischen Theologie als Terrain, auf dem das inhaltlich Gelehrte angepaßt wird. Ein Kommunikationstraining dieser Art, auch wenn es um die massenmediale Kommunikation geht, kommt immer zu spät. Es wird als fremd erfahren und schließt sich nicht den inhaltlichen Fächern an. Kurzum, ich denke, daß Kommunikation so wesentlich mit dem Theologisieren selbst verbunden ist, daß ein Student von Beginn seiner Studien an mit dieser Aufgabe vertraut gemacht werden muß.

Aus der Sicht der systematischen Theologie müssen einige Anmerkungen gemacht werden, die die Notwendigkeit einer guten und integrierten Kommunikationsausbildung illustrieren können. Theologie kann als Wissenschaft natürlich nicht bestehen ohne »Forschung«. Es geht hierbei um die Technik des Lesens, des Schreibens und Diskutierens, die zu jeder akademischen Ausbildung gehört. Die öffentliche Funktion des Theologen bedingt eine weitere Qualifikation. Ab und zu ist er oder sie aufgefordert zu intervenieren, einen Kommentar zu geben oder beizutragen zur öffentlichen Diskussion in den gedruckten, auditiven oder audiovisuellen Medien. Abgesehen von der Stellungnahme in solchen Diskussionen muß die Technik dieses Auftretens sorgfältig gelehrt werden. Außerdem bringt das Auftreten des Theologen in den Massenmedien eine spezifische Verantwortung mit sich, die thematisiert werden muß.

Mehr allgemein spielt die Frage nach einer guten Kommunikation als natürlicher Umgebung, als Kontext der Religion, eine Rolle. Theologie, und sicher systematische Theologie, ist eine Reflexion »a posteriori «, ein Nachdenken über den Glauben und seinen Ausdruck in der Vergangenheit und heute. Deshalb ist die Theologie auf die Glaubensgemeinschaft angewiesen, weil dort im doxologischen, das heißt symbolischen Sprechen (Das »First-Order «-Sprechen) das Material gefunden wird für die Theorieformung (Das »Second-Order «-Sprechen) im Dienst der Glaubenspraxis. 
Bei der Bibellesung, beim Studium der Tradition und im Dialog mit den aktuellen Gläubigen und ihrer Welt ist die Theologie abhängig von einer vorgegebenen Sprache. Für die Kommunikation ist dies ein sehr wichtiger Punkt. Es geht hier nicht in erster Linie um Sprache als konzeptuelles Instrument zur Wiedergabe der sogenannten objektiven Wirklichkeit, sondern um den Hintergrund erzählter Lebenserfahrung, in der die Theologie ihre reflexive Funktion ausübt.

In diesem Zusammenhang von Gemeinschaft und Sprache werden von der Theologie kommunikative Fertigkeiten erwartet. Zuallererst geht es darum, dialogisieren zu können. Es geht weiter um das Vermögen, Einsichten auch für andere begreifbar zu machen (zu objektivieren), um diese frei zur offenen Diskussion stellen zu können. Es ist außerdem eine große Beweglichkeit der Glaubenstradition im Spiel. Auf dem symbolisch-narrativen Niveau muß der Theologe den innerlichen Zusammenhang von Geschichten und ihren Symbolen einschätzen können, das Zentrum finden können, die Sprachtradition deuten können, die Form und Bedeutung gibt, kurzum die Lebensgeschichte von Menschen als Glaubenssprache beschreiben können. Vermag er das nicht, dann kann er seinen Beruf nicht ausüben. Die größte Schwierigkeit ergibt sich für interkulturelle und interdisziplinäre Gespräche. Kann man sehen und hören, kann man mitfühlen und mitdenken, bevor man selbst theologisches Gerede zu verkaufen anfängt?

Die Theologie muß auch kontextuell arbeiten können, d. h. teilnehmen können an den Gedankengängen und der Glaubenserfahrung der örtlichen Gemeinschaft. Und dennoch muß sie das Band mit der universellen Kirche festhalten können. Die Anbindung an die lokale Kirche ist wesentlich für das Theologisieren; ohne diese Verwurzelung gibt es keine Erfahrung, und es gibt keinen 'Vorgriff' der Interpretation.

Ein wichtiges kommunikatives Moment ist die Kenntnisnahme der Glaubenserfahrung und ihres Ausdruckes. Natürlich arbeitet die Theologie in Distanz zur Erfahrung, aber sie muß sich im klaren bleiben über die Tatsache, daß sie in der Wahl des Gegenstandes und von der Terminologie her in (verborgener) Abhängigkeit arbeitet. Theologie als Reflexion kann, wie konzeptuell und abstrakt sie ihr Denken auch ordnet, nicht isoliert werden von ihrer Erfahrungsumgebung.

Im allgemeinen kann man sagen, daß die theologische Kommunikation nicht allein eine Fertigkeit ist, um den internen systematischen Zusammenhang des Denkens herzustellen. Sie ist auch dazu da, daß sie ihrem eigenen Sprechen die richtige Sprachform gibt und den richtigen Moment wählt, um so die Menschen mit der Glaubenswirklichkeit zu verbinden. Der Theologe muß sprechen mit einer Sprache, die in dieser Welt verwurzelt ist und nicht in einem »krypto-religiösen « Jargon. Er muß eine Sprache verwenden, die allgemein ist und die Gleichzeitigkeit dieser Welt mit der anderen Welt zum Ausdruck bringen kann. Um unser sakramentales Modell noch einmal zu gebrauchen: Auch die Theologie muß in ihrem Sprechen (und in ihrem Schweigen) die Transparenz dieser Welt berücksichtigen. Erst wenn die Theologie diesen allgemeinen kommunikativen Auftrag erfüllt, ist sie auch in der Lage, einen integrierten Gebrauch von den Massenmedien zu machen.

Bei der Ausbildung der kirchlichen Mitarbeiter darf man die oben angegebenen interpersonalen Kommunikationsfertigkeiten ebenfalls nicht vernachlässigen zugunsten einer massenmedialen Ausbildung. Aus der Praxis heraus hat sich gezeigt, daß interpersonale und massenmediale Kommunikation komplementär zusammenstimmen 
müssen, will man mentale und strukturelle Veränderungen in der Kirche und in der Gesellschaft herbeiführen. Die Massenmedien haben in dieser Hinsicht kaum verändernden Einfluß, wenn und solange nicht die formellen und informellen Leiter der Kirche und Gesellschaft dies in die Hand nehmen.

Bei all dem möchte ich besonderen Wert legen aúf die Kommunikation vor und nach der theologischen Büroarbeit. Die theologische Reflexion arbeitet immer mit einer verallgemeinernden Konzeptualisierung nach dem Gespräch mit der Glaubenserfahrung der Schrift und Tradition und in Hinsicht auf die Konkretisierung ihrer Einsichten in die Glaubenspraxis des Alltags. Dies weist hin auf die Spannung zwischen dem Allgemeinen und dem Besonderen. Diese Spannung ist typisch für das theologische Arbeiten und setzt der abstrahierenden Wissenschaftlichkeit ihres Tuns Grenzen.

Die jüdischen und christlichen Traditionen haben ihre Gotteserfahrung immer mit konkreten Heilsgeschichten besonderer Menschen verbunden und den Gott Abrahams, Isaaks, Jacobs, Jesus', Augustinus' und Franzikus' etc. entdeckt. Anhand dieser Erzählungen lernen wir Gott kennen als »veränderlich «; das meint: in jeder konkretpartikulären Begegnung sich zeigend als derselbe und andere. Die Erzählungen sind nie vollkommen neu. Immer ist für den Hörer das Spiel mit der Möglichkeit des Erkennens und Wiedererkennens möglich. Daß sich der Zuhörer in bestimmten Geschichten zuhause fühlen kann, ist der Symbolik zu verdanken, von der die Geschichten Gebrauch machen, um das Herz des Menschen zu treffen. In der ersten Sprache des Glaubens sehen wir also ein spannungsgeladenes Zusammenspiel von partikulärer Erzählung und allgemeiner Symbolik.

Das ist das Verallgemeinern der partikulären Wirklichkeitsentdeckung auf perzeptuelle Art und damit nicht dasselbe wie die konzeptuelle Arbeitsweise in der wissenschaftlichen Theologie, die eine allgemeine Gültigkeit ihrer Einsichten verwirft aufgrund weitergehender Abstraktion. Es ist von Bedeutung, daß der Theologe diesen Unterschied kennt und daß er die partikulären Geschichten verstehen kann und den Zusammenhang der Symbole einzuschätzen vermag. Dann erst - wenn der »[kairos] «, der richtige Moment eintritt - kann die Theologie ein reflexives Instrumentarium anbieten, - zur Erhellung, zur Vertiefung, zur Verteidigung, zur Korrektur oder was auch immer im Gespräch nötig ist. Dann erst ist die Theologie nicht dominierend, nicht manipulierend, sondern dialogisch. Auf ihre eigene Weise gibt sie der Wirklichkeit Transparenz. Dann wirkt sie auch »sakramental « im Dienst der Kirche an der Welt.

\section{Anmerkung}

1 Der Beitrag ist eine überarbeitete Fassung aus: »MEDIA EN RELIGIEUZE COMMUNICATIE $\alpha$, Joan Hemels, Henk Hoekstra (Hrsg.) Hilversum, 1985, Seite 37 bis 48 . Übersetzung von der niederländischen in die deutsche Sprache: Franz Jussen. 


\section{SUMMARY}

The many connections between theology and communications are treated by the author of the article. He is proving that "communication $*$ is an essential component in modern concepts of revelation. Revelation ist communication itself but also aimed at "Communio and communication. This fact has to radiate on the Church Life and life in the Church. Ideally Church communication can transcend in form of a sacrament. An integrated and interdisciplinary introduction into communications should therefore be fostered to be part of the academic formation of theologans.

\section{RESUME}

Les relations entre théologie et communication sont abordées par l'auteur de l'article. Il montre que la *communication * est un élément essentiel du concept moderne de révélation. La révélation se manifeste comme communcation et vise la *communio et al communication. Ce fait doit pénétrer dans la vie de l'Eglise et vivre dans l'Eglise. Idéalement, la communication ecclésiale devrait comporter un caractère transcendantal à la manière d'un sacrement. C'est pourquoi la formation théologique devrait intégrer une introduction interdisciplinaire à la communication.

\section{RESUMEN}

El autor trata en su artículo, los múltiples puntos de contacto entre teología y comunicación. El autor demuestra de que »comunicación « es un elemento esencial en la idea moderna de la revelación. La revelación acontece como comunicación y tiene por objeto ncommunio y comunicación. Este hecho tiene que irradiarse a la práctica vivencial de la Iglesia. En el caso ideal, la comunicación eclesiástica puede conllevar como la forma de un sacramento, un carácter de remisión trascendental. En la instrucción de los teólogos, se tendrá que tener un cuidado intenso, de dar una introducción a la comunicación, que sea integrada e interdisciplinaria. 